through this gland, eventually arriving in the salivary duct from which they are excreted with the saliva during feeding of the aphid. Luteoviruses display a high degree of vector specificity at the various transmission barriers in the aphid. These well-developed specificities suggest an intimate association between a luteovirus and its vectors in which both surface domains of the viral capsid and sites or substances in the aphid are involved. The role of the viral capsid in conferring aphid transmissibility to a luteovirus has been convincingly demonstrated. Recently, aphid-derived components suggested to be involved in virus transmission were revealed by a protein blotbased virus overlay assay.

\section{Adaptation and application of immuno-PCR method to study the interactions of aphids and barley yellow dwarf luteoviruses. JQ Guo ${ }^{1}$, H Lapierre ${ }^{2}$, JP Moreau 1 (1 INRA, unité de zoologie; 2 INRA, unité de pathologie végétale, F-78026 Versailles cedex, France)}

The immuno-PCR method was adapted to amplify the French isolates of barley yellow dwarf, PAV, MAV and RPV luteoviruses in aphids. The sensitivity of this RT-PCR allowed for detection of $1 \mathrm{pg} / \mathrm{ml}$ of purified PAV virus preparation, and for a 128-fold dilution of a single viruliferous aphid (Rhopalosiphum padi). Conventional PCR could detect such single aphids with only an 8-fold dilution, even though the virus accumulated to near $1 \mathrm{ng} /$ aphid in a $5 \mathrm{~d}$ acquisition feeding on infected barley.

PAV virus was detected by immuno-PCR from the gut, salivary gland, hemolymph and legs, but not from the stylet and head of aphids of $R$ padi.

PAV, MAV and RPV viruses were recovered by immuno-PCR in the hemolymph of virus-carrying $R$ padi, Sitobion avenae, Metopolophium dirhodum, Diuraphis noxia, Myzus persicae, Aphis fabae and $A$ gossypii after a 2-d inoculation feeding on healthy barley.

In a serial transfer test, $R$ padi efficiently transmitted PAV and RPV, $S$ avenae efficiently transmitted PAV and MAV until the last transfer at $13 \mathrm{~d}$ on barley. The Md-Nord clone of $M$ dirhodum occasionally transmitted PAV and MAV, but not RPV, for up to 11 and $7 \mathrm{~d}$ transfers. Infrequent transmission of PAV was also found during the transfers of $7-11 \mathrm{~d}$ by $D$ noxia, but not MAV and RPV.

Virus content (405 OD value) in a batch of 10 aphids of $D$ noxia was much lower than that of $R$ padi, $S$ avenae and Md-Nord of $M$ dirhodum. The decrease of virus content in the aphids was biphasic following the serial transfers. The initial decrease occurred rapidly after the first transfer. In the second phase, the decrease trend differed among the aphid species, but without being bound to virus transmission. Immuno-PCR could detect PAV, MAV and RPV viruses in the aphids of 4 species until the last transfer at $13 \mathrm{~d}$, with the exceptions that for RPV in $D$ noxia and in the Md-Nord clone it was only to 7 and $11 \mathrm{~d}$ transfers, and for MAV in $D$ noxia to $11 \mathrm{~d}$ transfer.

Therefore, the aphid gut, as a barrier to the virion passage into the aphid's hemocoel, may not play a role of specific selection among these luteoviruses.

Intraspecific variations for transmission of BYDV-PAV and -MAV isolates by the aphids Sitobion avenae and Rhopalosiphum padi. E Sadeghi, CA Dedryver, S Tanguy, G Riault (INRA, laboratoire de zoologie, domaine de la Motte, F-35650 Le Rheu; 65, route de SaintBrieuc, F-35042 Rennes cedex, France)

\section{PAV transmission by S avenae}

Fifty Sitobion avenae clones were collected in January 1990 in the area of Rennes on wheat, barley and oats, and tested for their ability to transmit a local isolate of BYDV-PAV (PAV4) to barley (cv Express). The acquisition and inoculation conditions were standardized: $20^{\circ} \mathrm{C}$; L16/D8; $2 \mathrm{~d}$ acquisition; $5 \mathrm{~d}$ inoculation; 3 fourth instar larvae/test plant; 3 repetitions of 20 test plants/clone. Transmission percentages make a continuum from $3 \%$ for the worst transmitting clone to $92 \%$ for the best. There was however a non-negligable intraclonal variance. There was no non-transmitting clone but 5 clones were very bad vectors (less than 10\% transmission). Deep green clones transmit significantly less than clones of other colors. Clones collected on wheat and oats transmit significantly less than clones collected on barley.

These results incited us to study the possible role of aphid stylet activities on interclonal variations of PAV transmission (Prado and Tjallingii, 1994). Two very different clones were compared by the technique of EPG (electropenetrography: Tjallingii, 1978): Sa5 (3\% transmission) and Sa1 $(50 \%)$ on barley and wheat. Preliminary results show that clone Sa5 feeds very badly on barley, with long non-feeding periods, few and short 
sieve element phases (E1 and E2). Inversely, the good transmitting clone Sa1 feeds well on barley, with long sap ingestion periods, possibly allowing a better virus acquisition.

\section{PAV and MAV transmission \\ by Rhopalosiphum padi}

Twenty-five $R$ padi clones were tested for PAV and MAV transmission. They originated from different countries and continents (Europe, North America and North Africa) and exhibited different types of life-cycle (holocycle, anholocycle and androcycle). Concerning PAV4 transmission, we did not find any significant difference between clones, whatever the conditions of the experiments. The percentages of transmission were very high $(90-100 \%)$ in optimal conditions $(48 \mathrm{~h}$ acquisition $/ 5 \mathrm{~d}$ inoculation) and lower $(50-80 \%)$ when acquisition time or inoculation time were limiting $(6 \mathrm{~h})$. As regards MAV transmission, 2 isolates were compared, MAV2 (Le Rheu) and MAV11 (Versailles). MAV11 was only transmitted by 1 clone (Rp1: 3\% transmission) and MAV2 was transmitted by 2 clones, Rp3 (25\%) and Rp1 $(10 \%)$.

Prado E, Tjallingii WF (1994) Aphid activities during sieve element punctures. Entomol Exp Appl 72, 157-165

Tjallingii WF (1978) Electronic recording of penetration behaviour by aphids. Entomol Exp App/24, 521-530

Van der Heuvel JFJM, Verbeek M, van der Wilk $F$ (1994) Endosymbiotic bacteria associated with circulative transmission of potato leafroll virus by Myzus persicae. J Gen Vir 75, 2559-2565

Vector specificity of barley yellow dwarf (MAV, RPV) luteoviruses and virus regulation in aphids. JQ Guo 1, H Lapierre 2, JP Moreau 1 ( ${ }^{I N R A}$, unité de zoologie; 2 INRA, unité de pathologie végétale, F-78026 Versailles cedex, France)

The specificity and efficiency of barley yellow dwarf (MAV, RPV) luteoviruses were tested by using both 3 aphid clones of Rhopalosiphum padi $\mathrm{L}$ and Sitobion avenae Fabr in controlled conditions. All 3 clones of $R$ padi and $S$ avenae could transmit RPV and MAV, respectively, but vector efficiency was variable among them. Sa-R1 clone was a highly efficient vector (HEV), Sa-V clone was a moderately efficient vector (MEV) and SaR5 clone was a poorly efficient vector (PEV) within $S$ avenae to transmit MAV. Rp-M and Rp-R26 clones were the HEV and $\mathrm{Rp}-\mathrm{CH}$ clone was the PEV within $R$ padi to transmit RPV.

In the serial transmission test, the 3 clones of $S$ avenae and $2 \mathrm{HEV}$ clones of $R$ padi successively transmitted their respectively specific virus, MAV and RPV, until the last transfer at $13 \mathrm{~d}$ on barley, but the PEV Rp-CH clone transmitted RPV only up to $11 d$ transfer. After $5 d$ transfer, the vector efficiency declined obviously for all the clones. MAV and RPV contents in the aphids of 4 out of 6 clones decreased in triphase during the serial transfer, but the biphasic trend of MAV content and the monophasic trend of RPV content were involved in the virus regulation by the aphids of the PEV Rp-CH and the MEV Sa-V. In the triphasic trend, the initial decrease rapidly occurred after the first transfer, and then the virus content decreased slowly, but a relatively fast reduction appeared after $5 \mathrm{~d}$ transfer. In the biphasic trend, decrease rates of MAV content in the $\mathrm{Rp}-\mathrm{CH}$ and Sa-V were nearly identical in each transfer of the second phase. In the monophasic trend, the decrease rates of RPV content were almost similar in every transfer until after $5 \mathrm{~d}$ transfer when the virus was no longer detected by ELISA in a batch of 10 aphids.

Detection by immuno-PCR revealed that MAV was retained by the aphids of tested clones until the last transfer at $13 \mathrm{~d}$, except that $\mathrm{Rp}-\mathrm{CH}$ retained it up to $11 \mathrm{~d}$. RPV could be retained by the aphids of tested clones until the $13 \mathrm{~d}$ transfers, but $\mathrm{Rp}-\mathrm{CH}$ and Sa-V retained it only to 11 and $9 \mathrm{~d}$, respectively. Consequently, the reduction of MAV and RPV contents in the aphids was independent of the viruses that may be specifically transmitted during the serial transmission test. The moderate and poor vector efficiencies of $\mathrm{Sa}-\mathrm{V}$ and $\mathrm{Rp}-\mathrm{CH}$ clones were associated with the more rapid decrease of MAV and RPV contents in the aphids respectively. However, the poor vector efficiency of the Sa-R5 clone was not related to the reduction of virus content in the aphids. The selective barrier of accessory salivary glands (ASG) of aphids may determine vector specificity of MAV and RPV transmitted by $S$ avena and $R$ padi respectively, but clonal variations in vector efficiency within each species were associated with both the selective barrier of ASG and the capacity of virus retention by the aphids.

Assistance to long-distance transport between wheat yellow mosaic virus (WYMV) and soil-borne wheat mosaic virus (SBWMV) in bread wheat cultivars resistant to WYMV. 